\title{
Locating Adductor Canal and Quantifying the Median Effective Volume of Ropivacaine for Adductor Canal Block by Ultrasound
}

\author{
Chunguang Wang ${ }^{1}$, Zhiqiang Zhang ${ }^{2}$, Wenhai $\mathrm{Ma}^{3}$, Hui $\mathrm{Li}^{3}$ and Xinshuai $\mathrm{Du}^{4}$ \\ ${ }^{1}$ Department of Anesthesiology, The First Central Hospital of Baoding, Hebei, China \\ ${ }^{2}$ Department of Cardiothoracic Surgery, The First Central Hospital of Baoding, Hebei, China \\ ${ }^{3}$ Department of Orthopaedic, The First Central Hospital of Baoding, Hebei, China \\ ${ }^{4}$ Department of Medical Ultrasonics, The First Central Hospital of Baoding, Hebei, China
}

\begin{abstract}
Objectives: To identify the precise position of adductor canal; and to determine median effective volume ( $\mathrm{EV}_{50}$ ) for adductor canal block (ACB) by ultrasound.
\end{abstract}

Study Design: Observational study.

Place and Duration of Study: Department of Anesthesiology, the First Central Hospital of Baoding, China from January 2019 to December 2020.

Methodology: One hundred and ninety subjects were recruited for mapping, the precise location of adductor canal. Twenty-four patients were scheduled to study the $\mathrm{EV}_{50}$ of ropivacaine. Ultrasound was used to identify the anatomical structure of femoral triangle as well as the cephalad and caudad end of adductor canal. By using the up-and-down method to study the effect of nerve block, the $\mathrm{EV}_{50}$ of $0.5 \%$ ropivacaine on $\mathrm{ACB}$ was quantified.

Results: The femoral triangle, the cephalad, and caudad end of adductor canal were clearly shown in the ultrasound images of all subjects. The midpoint between anterior superior iliac spine and the superior margin of patella is in femoral triangle. The cephalad edge of adductor canal is distal to the midpoint between anterior superior iliac spine and the superior margin of patella. The midpoint between the femoral trochanter and the superior margin of patella is in the adductor canal. $\mathrm{EV}_{50}$ of $0.5 \%$ Ropivacaine for ACB is $10.79 \mathrm{ml}(95 \% \mathrm{Cl}, 10.10-11.52 \mathrm{ml})$.

Conclusion: The midpoint between the femoral trochanter and the superior margin of patella is in the adductor canal. $\mathrm{EV}_{50}$ of $0.5 \%$ ropivacaine for $\mathrm{ACB}$ is $10.79 \mathrm{ml}(95 \% \mathrm{Cl}, 10.10-11.52 \mathrm{ml})$.

Key Words: Adductor canal, Location, Ultrasound, Median effective volume, Nerve block.

How to cite this article: Wang C, Zhang Z, Ma W, Li H, Du X. Locating Adductor Canal and Quantifying the Median Effective Volume of Ropivacaine for Adductor Canal Block by Ultrasound. J Coll Physicians Surg Pak 2021; 31(10):1143-1147.

\section{INTRODUCTION}

Knee osteoarthritis, a chronic degenerative disease of knee joint, greatly reduces the quality of life of the aged. Total knee arthroplasty (TKA) is one of the most effective treatments that restores function of knee joint for patients with knee osteoarthritis at the end-stage. Unfortunately, according to statistics, there were about $60 \%$ of patients suffering from severe pain and up to $30 \%$ suffer from moderate pain following TKA. ${ }^{1}$

Correspondence to: Chunguang Wang, Department of Anesthesiology, The First Central Hospital of Baoding, Hebei, China

E-mail: wangchunguang@163.com

Received: May 19, 2021; Revised: August 03, 2021;

Accepted: September 13, 2021

DOI: https://doi.org/10.29271/jcpsp.2021.10.1143
Nerve block is frequently used by the anesthesiologists to gain better postoperative pain control after surgery. It is critical for anesthesiologist to find effective perioperative anesthetic dose and precise location of target nerve for patients following TKA.

Postoperative pain can be improved by adductor canal block $(A C B)$, which is an alternative to femoral nerve block. ${ }^{2-4}$ Currently, the most common needle insertion site of ACB is the midpoint between the anterior superior iliac spine (ASIS) and superior margin of patella..$^{4-8}$ Unfortunately, needle insertion of $\mathrm{ACB}$ at the well-known position, might cause quadriceps paralysis. ${ }^{9,10}$ In a recent study, Wong et al. found that the injection at this well-known position was a femoral triangle block actually. ${ }^{11}$ The motor branches of femoral nerve can be affected accidently by local anesthetics. Bendtsen et al. considered that the needle insertion site of ACB should be defined as the midpoint between the throchanter and the superior margin of patella. ${ }^{12}$ It is necessary to make map of the adductor canal for anesthesiologist to 
achieve ACB successfully. Based on the anatomical evidences, the cephalad end of adductor canal was defined as the intersection of the medial edge of the sartorius muscle and the adductor longus muscle. ${ }^{13}$ The caudad end of adductor canal was defined as adductor hiatus. ${ }^{13}$ Ultrasound was used to identify the accurate position of adductor canal in Chinese subjects in this study.

In addition to the precise location of the injection, the quality of nerve block is affected by the volume of local anesthetic. Excess injection in adductor canal may induce a spread of local anesthetics toward into femoral triangle. ${ }^{11-12}$

Therefore, it is important to find the optimal volume of local anesthetics for ACB, providing adequate analgesia and avoiding weakened muscles strength. Therefore, the aim of this study was to seek ultrasound guidance for location and determining the $\mathrm{EV}_{50}$ of $0.5 \%$ ropivacaine for true $A C B$.

\section{METHODOLOGY}

This prospective observational study and Dixon and Massey upand-down sequential trial were complied with the Declaration of Helsinki. Ethical clearance was granted by the Ethical Committee of The First Central Hospital of Baoding ([2018] 038) and written informed consents were obtained from all subjects. Subjects undergoing TKA were enrolled into this research from January 2019 to December 2020 in The First Central Hospital of Baoding. Subjects with a history of lower extremity surgery before TKA, deformity, or complication of lower extremity, contraindication of nerve block, diabetes, allergy to ropivacaine, and application of analgesics more than four weeks (administration of morphine equivalents by oral or intramuscularorintravenous), renal and hepatic dysfunction and uncooperative patient for assessments, were excluded. One hundred and ninety subjects, scheduled for total knee arthroplasty, were recruited for determination the exact position of adductor canal and scheduled to apply a true ACB guided by ultrasound for evaluating the analgesic effect and influence on quadriceps muscle strength. Twenty-four patients, scheduled for total knee arthroplasty, were scheduled to receive a true ACB guided by ultrasound for studying the $\mathrm{EV}_{50}$ of $0.5 \%$ ropivacaine.

The subjects laid on operation table in a supine position. The ASIS, the throchanter, the midpoint between ASIS and the superior margin of patella, the midpoint between the throchanter and the superior margin of patella were marked as in Figure $1 \mathrm{~A}$. The ultrasound probe was placed on the surface of inguinal crease at anteromedial and proximal part of the thigh. Scanning along the femoral artery, the structure of femoral triangle could be seen gradually (Figure $1 \mathrm{~B}){ }^{13}$ The probe was slid distally to the cephalad end of adductor canal, which was the intersection of the medial edge of the sartorius muscle and the adductor longus muscle (Figure 1C). ${ }^{14}$ Adductor canal was surrounded by the sartorius muscle, the vastus medialis muscle and the adductor longus muscle (Figure 1D). The probe was slide distally further until the femoral artery passed the adductor hiatus to become popliteal vessels (FigurelE). The adductor hiatus, also called the caudad end of adductor canal, ${ }^{15}$ was marked (Figure 1A). The distance from the superior margin of patella to the midpoint between ASIS and the superior margin of patella, the midpoint between the throchanter and the superior margin of patella, the cephalad end of adductor canal, the caudad end of adductor canal, and the length of adductor canal were measured.

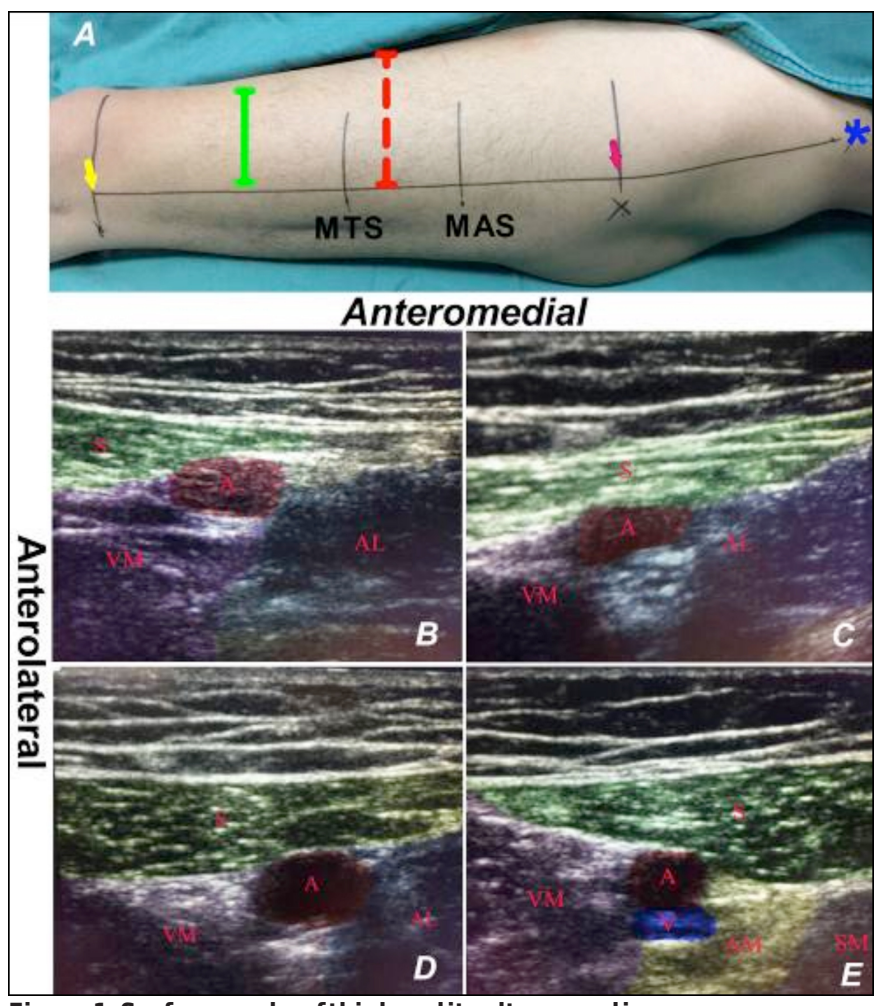

Figure 1: Surface marks of thigh and its ultrasound images.

$1 \mathrm{~A}$ indicates, the blue asterisk indicates anterior superior iliac spine (ASIS); the pink arrow indicates the the throchanter; MAS indicates the midpoint of distance from ASIS to the superior margin of patella; MTS indicates the midpoint between thethrochanterand thesuperiormargin of patella; theyellow arrowindicatesthe superior margin of patella; the red dotted line indicates the cephalad end of adductor canal and the green solid line indicates the caudad end of adductor canal. 1B indicates ultrasound image of femoral tringle, at the section of MAS in figure $1 \mathrm{~A}$. 1C indicates ultrasound image of the cephalad end of adductor canal, at the section of red dotted line in figure 1A. 1D indicates ultrasound image of adductor canal, at the section of MTS in figure 1A. 1E indicates ultrasound image of the caudad end of adductor canal, at the section of green solid line. In 1A, S represents sartorius muscle, VM represents medialis muscle, AL represents adductor longus muscle, AM represents adductor magnus muscle, SM represents semimembranousus muscle, A represents femoral artery, and $V$ representsfemoralvein.

All patients were not given any premedication and laid on operation table supine. The ultrasound probe was placed on the anteromedial of the thigh; and the structure of adductor canal was identified by ultrasound. A needle, guided by ultrasound, was inserted into the triangular echo region lateral to femoral artery. The diffusion of local anesthetics in the triangular echo region was shown in the ultrasound image after injection of $0.5 \%$ Ropivacaine.

Cutaneous sensation innervated by saphenous nerve (the anteromedial aspect of knee, shin area) were assessed 30 minutes after injection. The complete sensory block was defined as the absence of pinprick sensation in the area inner- 
vated by saphenous never. The criteria for sensory evaluation as 0 (totally loss of sensation), 1 (loss of touch sensation but not pinprick sensation), and 2 (no loss of sensation, similar to contralateralside). ${ }^{16}$ Thequadriceps musclestrength was evaluated right after sensory assessment. The evaluation of muscle strength referred to the manual muscle testing reported by Wintz. ${ }^{17}$ Postoperative pain and quadriceps strength were measured at 4, 8, 12 and 24 hours after TKA.

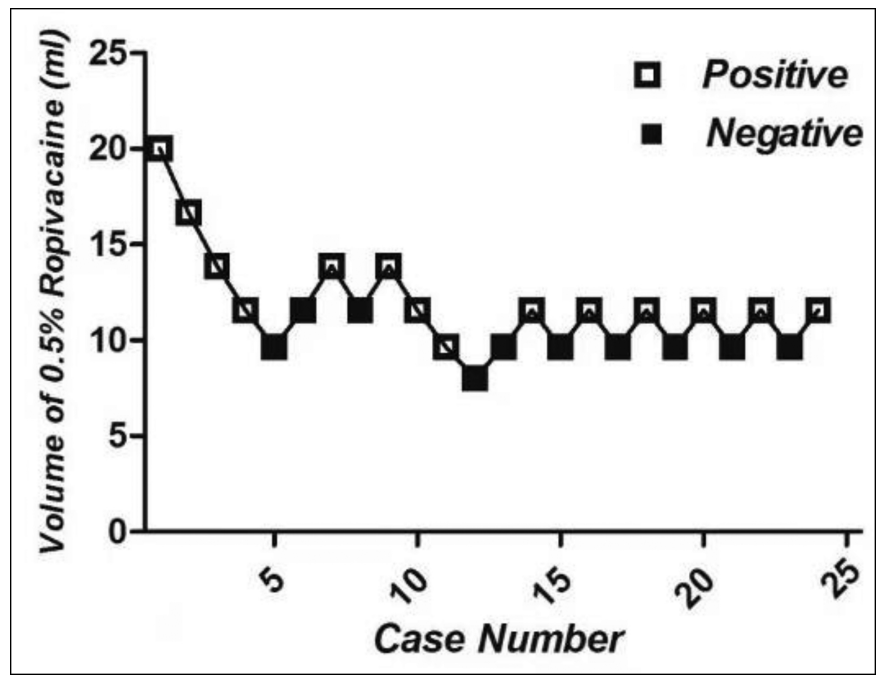

Figure 2: Sequential responses to up-and-down volume of $0.5 \%$ Ropivacaine for adductor canal block.

Hollow square indicates positive response, meaning sensory block completely; blacksquareindicatesnegativeresponse, meaning sensory blockincompletely.

For studying the $\mathrm{EV}_{50}$ of $0.5 \%$ ropivacaine, $20 \mathrm{ml}$ of $0.5 \%$ Ropivacaine was chosen as the initial dose based on the previous research. ${ }^{18}$ The dose interval was set at 1:1.2 according to the response of the previous patient (complete or incomplete block) based on Dixon's up-and-down method. ${ }^{19}$ The research was ended by eight consecutive up-and-down sequences.

SPSS version 17.0 was used for statistical analysis. Demographics data such as age, weight and height and measurement data of adductor canal were shown as mean \pm SD. Data were shown as median (IQR) for non-normally distributed variables, such as VAS score and quadriceps muscle strength score. The median effective volume of $0.5 \%$ Ropivacaine was determined by the up-and-down sequence method of Dixon and Massey. ${ }^{19} P<0.05$ was considered statistically significant.

\section{RESULTS}

Demographics of 190 subjects, including 98 males and 92 females, 103 left and 87 right lower limb, with mean age of 68.6 \pm 6.4 years, weight of $63.7 \pm 8.9 \mathrm{Kg}$ and height $165.3 \pm 9.8 \mathrm{~cm}$, recruited for determination the exact position of adductor canal. Ultrasound images of femoral triangle, the cephalad and the caudad end of adductor canal were clearly shown in all subjects (Figure 1). For all subjects, the cephalad end of adductor canal was distal to the midpoint between ASIS and the superior margin of patella. In 182/190 subjects, the cephalad end of adductor canal was proximal to the midpoint between the throchanter and the superior margin of patella; whereas, the rest 8/190 subjects were distal. The distance from the superior margin of patella to the midpoint between ASIS and the superior margin of patella, the midpoint between the throchanter and the superior margin of patella, the cephalad end of adductor canal, the caudad end of adductor canal and the length of adductor canal are shown in Table I. The VAS scores were no more than 4 and the quadriceps strength were more than 4 within 24 hours after TKA in most subjects (Table II).

Twenty-four patients, including 15 males and 9 females, with mean age of $61.3 \pm 4.3$ years, weight of $69.3 \pm 6.6 \mathrm{Kg}$ and height $172.2 \pm 5.1 \mathrm{~cm}$, were enrolled for studying the median effective volume of $0.5 \%$ ropivacaine. The sequences of positive and negative responses were presented in Figure 2. Fourteen and 10 patients showed complete and incomplete sensory block, respectively. The median effective volume of $0.5 \%$ ropivacaine for ACB was $10.79 \mathrm{ml}(95 \% \mathrm{Cl}, 10.10-11.52 \mathrm{ml})$.

Table I: Measurement data of adductorcanal $(n=190)$.

\begin{tabular}{|c|c|}
\hline Distance to MAS, cm & $21.5 \pm 2.6$ \\
\hline Distance to MTS, cm & $15.1 \pm 1.8$ \\
\hline Distance to CAC, cm & $17.5 \pm 1.9$ \\
\hline Distance to CEAC, cm & $9.2 \pm 1.1$ \\
\hline Length of $\mathrm{AC}, \mathrm{cm}$ & $8.3 \pm 1.1$ \\
\hline \multicolumn{2}{|c|}{$\begin{array}{l}\text { Data are presented as mean } \pm \text { standard deviation. MAS: the midpoint } \\
\text { from anterior superior iliac spine to the superior margin of patella; MTS: } \\
\text { the midpoint from the throchanter to the superior margin of patella; CAC: } \\
\text { the cephalad end of adductor canal; CEAC: the caudad end of adductor } \\
\text { canal; Distance to MAS: the distance from MAS to the superior margin of } \\
\text { patella; Distance to MTS: the distance from MTS to the superior margin of } \\
\text { patella; Distance to CAC: the distance from CAC to the superior margin of } \\
\text { patella; Distance to CEAC: the distance from CEAC to the superior margin } \\
\text { of patella; Length of AC: the distance from CAC to CEAC. }\end{array}$} \\
\hline
\end{tabular}

Table II: VAS and quadriceps strength $(n=190)$.

\begin{tabular}{|l|c|}
\hline VAS score & $1(0-1)$ \\
\hline $4 \mathrm{~h}$ at rest & $1(1-2)$ \\
\hline 8h at rest & $2(1-3)$ \\
\hline $12 \mathrm{~h}$ at rest & $2(2-3)$ \\
\hline 24h at rest & $2(0-2)$ \\
\hline 4h during active flexion & $3(1-3)$ \\
\hline 8h during active flexion & $3(2-3)$ \\
\hline $12 \mathrm{~h}$ during active flexion & $3(2-3)$ \\
\hline $24 \mathrm{~h}$ during active flexion & $4(3-5)$ \\
\hline Quadriceps strength & $4(3-5)$ \\
\hline 30 min after ACB & $5(4-5)$ \\
\hline $4 \mathrm{~h}$ & $5(4-6)$ \\
\hline 8h & $6(5-6)$ \\
\hline 12h & \\
\hline 24h & \\
\hline Data are expressed as median (IQR). ACB: Adductor canal block. \\
\hline
\end{tabular}

\section{DISCUSSION}

$A C B$, as a component of multimodal analgesia, is widely used to provide adequate postoperative pain control and early rehabilitation after TKA. ${ }^{8}$ It is common knowledge that peripheral nerve block can be achieved successfully relying on the precise location of the nerve. However, there are some disputes about the accu- 
rate position of adductor canal in present days. The well-known approach for performing $A C B$, originally described as the midpoint of ASIS and the superior margin of patella, was thought to be in femoral triangle rather than adductor canal. ${ }^{11}$ Anatomically, the cephalad end of adductor canal is defined as the intersection of the medial edge of the sartorius muscle and the adductor longus muscle. The caudad end of adductor canal is defined as adductor hiatus. ${ }^{13}$ Ultrasound can be used to identify the location of adductor canal by identifying the musculoskeletal structure. In the present study, the cephalad end of adductor canal was distal to the midpoint of ASIS and the superior margin of patella in all 190 Chinese subjects, which is consistent with the previously reported results by Wong et al. and Anagnostopoulou et al. ${ }^{11,20}$ The results can explain the weakness of quadriceps strength after single or continuous ACB. However, there was a difference showed by Anagnostopoulou et al. that the cephalad end of adductor canal was located proximal to the midpoint between ASIS and the superior margin of patella in 4 of 17 dissected specimens. The methodological differences between cadaver dissection and ultrasound may be a possible explanation.

In this research, it was also found that the midpoint between the throchanter and the superior margin of patella was in the adductor canal in 182 Chinese subjects. This result indicated that the midpoint between the throchanter and the superior margin of patella may be the appropriate puncture position for ACB. However, there were 8 cases that the midpoint between the throchanter and the superior margin of patella was proximal to the cephalad end of adductor canal, which might due to individual anatomical variations. Therefore, a combination of surface and sonographic landmarks can assist the anesthesiologists to target correct anatomical location and achieve complete nerve blockage. Moreover, the VAS scores were no more than 4 and the quadriceps strength were more than 4 within 24 hours after TKA in this study, that reveals the true ACB, at the midpoint between the throchanter and the superiormargin of patella, can improve pain withoutaffecting motor function.

In addition to the precise location of nerve, the quality of nerve block was also affected by the volume and concentration of local anesthetic. Owing to the musculoaponeurotic tunnel structure, the optimal volume of ropivacaine for ACB should be enough for fully filling the adductor canal but avoid proximal spread to the femoral triangle. In this present research, the median effective volume of $0.5 \%$ ropivacaine was $10.79 \mathrm{ml}(95 \% \mathrm{Cl}, 10.10-11.52$ $\mathrm{ml}$ ), consistent with the data reported by Tao et al. and Montgeomery et al. ${ }^{8,21}$ Although similar volumes were obtained, the needle insertion site in Tao et al.'s report should be located in femoral triangle rather than adductor canal based on our above results. As a result, local anesthetics have a higher chance to diffusing to the femoral nerve and blocking the branches. This study uses a lower injection site located right in adductor canal. Due to the limitation of musculoaponeurotic tunnel structure of adductor canal, the probability of lateral spread decreases. A low volume of local anesthetics could fully diffuse within the whole adductor canal and block the saphenous nerve in this study. Moreover, the scores of quadriceps strength at $30 \mathrm{~min}$ after ACB was $4 .^{3-5}$ However, it is a fact that excessive local anesthetic's spread into femoral triangle, ${ }^{11-12}$ may increase the risk of failing in certain particular conditions.

There are some limitations in this study. First, the spread range of ropivacaine was assessed only by the blockade of sensory and motor. Combined with contrast material, imaging technology could have helped ascertain the extent of spread. Second, unlike surgical anesthesia, postoperative analgesia does not require a complete blockade. The block's success was defined as a total sensory blockade in the present study. Therefore, some of the patients defined as block failure would have received analgesia in clinical setting. Third, it is a methodological drawback that 10 patients experienced non-satisfactory nerve block by the Dixon's up-and-down method. A catheter, as analgesic remedy, was placed to minimise ethical concerns in the present study.

\section{CONCLUSION}

The midpoint between ASIS and the superior margin of patella was in femoral triangle. The cephalad edge of adductor canal was distal to the midpoint between ASIS and the superior margin of patella. The midpoint between the trochanter and the superior margin of patella was in the adductor canal. Moreover, the $\mathrm{EV}_{50}$ of $0.5 \%$ ropivacaine for $\mathrm{ACB}$ was $10.79 \mathrm{ml}(95 \% \mathrm{Cl}$, 10.10-11.52 ml).

\section{ETHICALAPPROVAL:}

Ethics clearance was granted by the Ethical Committee of The First Central Hospital ofBaoding ([2018]038).

\section{PATIENTS' CONSENT:}

All subjects signed an informed consentform.

\section{CONFLICT OF INTEREST:}

The authors declared no conflict of interest.

\section{AUTHORS' CONTRIBUTION:}

CW: Design the study, collected and analysed data, drafted and revised the paper.

ZZ: Drafted the paperand revised it.

WM, HL: Work for acquisition, analysis of data for this study. $X D$ : Work for acquisition of data for this study.

\section{REFERENCES}

1. Hebl JR, Dilger JA, Byer DE, Kopp SL, Stevens SR, Pagnano $\mathrm{MW}$, et al. A pre-emptive multimodal pathway featuring perpheral nerve block improves perioperative outcomes after major orthopedic surgery. Reg Anesth Pain Med 2008; 33(6):510-7.

2. Leung P, Dicherson DM, Denduluri SK, Mohammed MK, Lu $M$, Anitescu $M$, et al. Postoperative continuous adductor canal block for total knee arthroplasty improves pain and functional recovery: A randomised controlled clinical trial. J Clin Anesth 2018; 49:46-52. doi: 10.1016/j.jclinane.2018. 06.004.

3. Jaeger P, Nielsen ZJ, Henningsen $M H$, Hilsted KL, Mathiesen O, Dahl JB. Adductor canal block versus femoral nerve block 
and quadriceps strength: a randomized, double-blind, placebo-controlled, crossover study in healthy volunteers. Anesthesiology 2013; 118(2): 409-15. doi: 10.1097/ALN. 0b013e318279fa0b.

4. Henshaw DS, Jaffe JD, Reynolds JW, Dobson S, Russell GB, Weller RS. An evalution ultrasound-guided adductor canan blockade for postoperative analgesia after medial unicondylar knee arthroplasty. Anesth Analg 2016; 122(4):1192-201. doi: 10.1213/ANE.0000000000001162.

5. Grevstad U, Mathiesen O, Valentiner LS, Jaeger P, Hilsted $\mathrm{KL}$, Dahl JB. Efeect of adductor canal block versus feormal nerve block on quadriceps strength, mobilization, and pain after total knee arthroplasty: A randomised, blined study. Reg Anesth Pain Med 2015; 40(1):3-10. doi: 10.1097/ AAP.0000000000000169.

6. Tao Y, Zheng SQ, Xu T, Wang G, Wang Y, Wu AS, et al. Median effective volume of Ropivacaine $0.5 \%$ for ultrasound-guided adductor canal block. J Int Med Res 2018; 46(10): 4207-13. doi: 10.1177/0300060518791685.

7. Ibrahim AS, Aly MG, Farrag WS, Gad El-Rab NA, Said HG, Saad AH. Ultrosound-guided adductor canal block after arthroscopic anterior cruciate ligament reconstrution: Effect of adding dexamethasone to bupivacaine, a randomised controlled trial. Eur J Pain 2019; 23(1): 135-41. doi: 10.1002/ejp.1292.

8. Kampitak W, Tanavalee A, Ngarmukos S, Amarase C. Opioid-sparing analgesia and enhanced recovery after total knee arthroplasty using combined triple nerce blocks with local infiltration analgesia. J Arthroplasty 2019; 34(2): 295-302. doi: 10.1016/j.arth.2018.10.009.

9. Chen J, Lesser JB, Hadzic A, Reiss W, Resta-Flarer F. Adductor canal block can result in motor block of the quadriceps muscle. Reg Anesth Pain Med 2014; 39(2):170-1. doi: 10.1097/AAP.0000000000000053.

10. Veal C, Auyong DB, Hanson NA, Allen CJ, Strodtbeck W. Delayed quariceps weakness after continuous sdductor canal block for total Inee arthroplasty: A case report. Acta Anaesthesiol Scand 2014; 58(3):362-4. doi: 10.1111/ aas. 12244

11. Wong WY, Bjorn S, Strid JM, Borglum J, Bendtsen TF. Defining the location of the adductor canal using ultrasound. Reg Anesth Pain Med 2017; 42(2):241-5. doi:
10.1097/AAP.0000000000000539.

12. Bendtsen TF, Moriggl B, Chan V, Pedersen EM, Borglum J. Redefining the adductor canal block. Reg Anesth Pain Med 2014; 39(5):442-3. doi: 10.1097/AAP.0000000000000119.

13. Ghassemi J, Gray AT: The adductor canal block. In: Jankovic $D$, Peng $P$, editors. Regional nerve blocks in anesthesia and pain therapy: Traditional and ultrasound-guided techniques. $4^{\text {th }}$ ed. New York: Springer International Publishing Inc; 2015; p. 843-51.

14. Bendtsen TF, Moriggl B, Chan V, Borglum J. Basic topography of the saphenous nerve in the femoral triangle and the adductor canal. Reg Anesth Pain Med 2015; 40(4):391-2. doi: 10.1097/AAP.0000000000000261.

15. Saranteas T, Anagnostis G, Paraskeuopoulos T, Koulais D, Kokkalis Z, Nakou M, et al. Anatomy and clinical implications of the ultrasound-guided subsartorial saphenous block. Reg Anesth Pain Med 2011; 36(4):399-402. doi: 10.1097/AAP.0b013e318220f172.

16. Wang CG, Yang JP, Hu CQ, Ding YL. The effect of adding dexmedetomidine to ropivacaine for lumbar plexus and sciatic nerve block. Int J Clin Exp Med 2016; 9:14198-204.

17. Wintz MM. Variations in current manual muscle testing. Phys Ther Rev 1959; 39(7):466-75. doi: 10.1093/ptj/ 39.7.466.

18. Jaeger $\mathrm{P}$, Jenstrup MT, Lund J, Siersma V, Brondum V, Hilsted $\mathrm{KL}$, et al. Optimal volume of local anaesthetic for adductor canal block: Using the continual reassessment method to estimate ED95. BrJ Anaesth 2015; 115(6):920-6. doi: 10.1093/bja/aev362.

19. Dixon WJ, Massey FJ. Introduction to statistical analysis. $4^{\text {th }}$ ed. New York: McGraw-Hill 1983; 428-39.

20. Anagnostopoulou S, Anagnostis G, Saranteas T, Mavrogenis AF, Paraskeuopoulos T. Saphenous and infrapatellar nerves at the adductor canal: Anatomy and implications in regional anesthesia. Orthopedics 2016; 39(2):259-62. doi: 10.3928/ 01477447-20160129-03.

21. Montgomery SH, Shamji CY, Yi GS, Yarnold CH, Bell SC, Schwarz SK. Effect of nerver stimulation use on the success rate of ultrasound-guided subsartorial saphenous nerver block: a randomised controlled trial. Reg Anesth Pain Med 2017; 42(1):25-31. doi: 10.1097/AAP.0000000000000522. 